\title{
Varroa destructor mites regularly generate ultra-short, high magnitude vibrational pulses
}

\author{
Harriet Hall1, Martin Bencsik1,*, Michael I. Newton¹, David Chandler², Gillian Prince², \\ and Scott Dwyer ${ }^{2}$
}

\author{
1 School of Science and Technology, Nottingham Trent University, Clifton Lane, NG11 8NS, Nottingham, United Kingdom \\ 2 School of Life Sciences, University of Warwick, Wellesbourne Campus, CV35 9EF, UK \\ * Corresponding author: martin.bencsik@ntu.ac.uk
}

With 6 figures

\begin{abstract}
The ectoparasitic mite Varroa destructor is considered one of the greatest threats to the honeybee Apis mellifera. To successfully manage mite populations residing in the colony, beekeepers must stay informed of infestation levels in their apiaries. The remote, non-destructive detection of Varroa mites in honeybee hives would therefore be highly desirable. Here we show that an ultra-sensitive $(1000 \mathrm{mV} / \mathrm{g})$ accelerometer can detect vibrational waveforms originating from one individual mite. We further focus on a commonly observed pulsing behaviour never before described, characterising its physical features, periodicity and strength. The spectral features of the detected pulses strongly depend on the substrate on which they are produced. The characteristics of the vibrational pulse, particularly its repeatability and strength, indicate that mite vibrations could be successfully detected in a fully populated honeybee hive. These features, combined with the remarkably high varroa muscular power output (up to $810 \mathrm{nW}$ ) indicate that this pulse may be functional for the mite. Our results uncover an exciting novel behaviour and provide a foundation for the remote detection of mites in beehives using vibration capture.
\end{abstract}

Keywords: varroa, vibration, biotremology, honeybee, behaviour

\section{Introduction}

The Varroa destructor mite (Anderson \& Trueman 2000) is described as the most influential ectoparasite of the European honeybee, Apis mellifera, in terms of its destructive impact (Rosenkranz et al. 2010; Evans \& Cook 2018). Bee health and development is negatively affected by the mite feeding on both adult bees and larvae, particularly as by doing so, it acts as a vector of viral diseases (Kevan et al. 2006; Chen \& Siede 2007). Evidence has recently been found to demonstrate that mites feed primarily on the fat body of adult bees, which is an important tissue necessary for protein synthesis, pesticide detoxification and successful overwintering of colonies (Ramsey et al. 2019). Mite and bee are closely linked because the parasite lacks a free-living stage, instead adopting a two-phase life cycle: phoretic (on adult bees) and reproductive (within sealed brood cells). The adult bees carry a phoretic mite towards a brood cell, and the mite enters it a short time before the cell is capped in order to begin its reproductive phase (Rosenkranz 2010; Nazzi \& Le Conte 2016). Once in the cell, mite reproduction appears to be synchronised with the development of bee larvae, regulated by the production of larval signals (Frey et al. 2013).

\subsection{The importance of detecting and monitoring Varroa mites in honeybee hives}

Varroa infestation can lead to the complete destruction of colonies, particularly over the winter months as high mite levels in the autumn impact upon bee lifespan. This in turn affects the bees' ability to transition from a winter bee into a summer bee, which is critical for colony success moving into the warmer months (Le Conte et al. 2010). The phenomenon known as Colony Collapse Disorder (CCD) is also thought to be linked to Varroa presence in a hive, as mites are the cause of many adverse reactions in honeybees. It is the interaction between these mite-related effects, combined with other stressors such as pesticides that is accepted to lead a colony to CCD (Le Conte et al. 2010). Beekeepers therefore need to stay on top of Varroa mite infestation in their apiaries. Many beekeepers treat preventatively, regardless of mite population size (Lee et al. 2010). However, 'blind' use of chemical control can be detrimental without knowing the full extent of 
the infestation level and whether treatment is truly needed at that time. This is due to the adverse effects of acaricides on honeybee processes and functions (Tihelka 2018), and mite resistance to chemical control, which has been documented around the world (Milani 1999; Martin 2004). This could be avoided through infestation checks and the use of alternative or rotated treatment types (Milani 1999; Pettis 2004). Regular monitoring is particularly useful to identify trends in the mite population (Gregorc \& Sampson 2019), so that the mite control treatment can be applied at the correct time, if at all needed. However, at present, the monitoring methods available to beekeepers and honeybee/Varroa mite researchers are time-consuming, require physical visitation to hives and can be disruptive to the colony.

Counting the natural mite-fall to produce a whole colony infestation estimate, for example, is inaccurate unless it is done daily over a prolonged period (Bienkowska \& Konopacka 2001; Pietropaoli et al. 2021), which leads to regular visitation requirements. Other techniques such as sugar shake, washing and brood sampling require the removal and/or destruction of adult bees and brood (Dietemann et al. 2013; Barlow \& Fell 2006). Although the sugar shake method allows bees to be placed back in the hive, there is still colony disruption caused, as well as inaccuracies that can be caused by sugar quality, temperature or humidity (Fakimzadeh et al. 2011; Gregorc et al. 2017).

\subsection{Remote Varroa monitoring techniques}

There has been a recent move towards investigation into remote monitoring techniques for honeybee colonies, including Varroa detection. Improvements to reduce the number of hive visitations and negative impacts upon the colonies can be only be a positive step. Currently, the use of gas sensors (otherwise known as electronic noses, capable of identifying complex odours (Bąk et al. 2020)) and video for detecting mite presence in hives are being explored. At present, these sensors are being tested for their ability to discriminate between healthy and mite-infested colony odours (Bąk et al. 2020; Szczurek et al. 2020a; Szczurek et al. 2020b; Konig 2021), and in most cases these can be inserted into a hive and left to carry out continuous measurements that can be accessed remotely (Szczurek et al. 2020a; Szczurek et al. 2020b; Konig 2021). However, they are at risk of being propolised. In terms of video detection, various image processing techniques and training software have been employed to identify the presence of a mite in video recordings of the adult bees or within brood cells (Ramirez et al. 2012; Elizondo et al. 2013; Chazette et al. 2016; Bjerge et al. 2019; Bilik et al. 2021). This work is currently in a prototype phase with some limitations, including video clarity affecting the chance of a successful detection (Elizondo et al. 2013) and the requirement for powerful hardware to carry out complex computations (Bilik et al. 2021). In the field, videos can only be recorded at the hive entrance (Bjerge et al. 2019), high- lighting another flaw as this type of monitoring presently cannot take place within the darkness of the hive.

Here, we employ ultra-sensitive $(1000 \mathrm{mV} / \mathrm{g})$ accelerometer sensors to capture vibrations produced by mite individuals. This technology has previously been employed by our research group for the remote detection of various individual honeybee vibratory signals, as well as providing vibration information on the status of the colony as a whole (Bencsik et al. 2015; Ramsey et al. 2018; Ramsey et al. 2020). The sensors are secured to the comb at the centre of the hive and can be left for unlimited, prolonged periods to continuously monitor and record the vibrations captured within the colony, irrespective of propolis/wax coating or darkness levels. In this present work we establish whether mite vibrations can be successfully detected with this technology, with future implications for its use within honeybee hives to remotely and continuously monitor Varroa levels. In doing so, we also present a new Varroa behaviour.

\section{Methods and materials}

\subsection{Mite collection}

Measurements were undertaken at Nottingham Trent University between August 2019 and December 2019. Live Varroa mites were identified and collected daily from the baseboard of a colony of Apis mellifera. Mites were immediately taken to the laboratory for use within one hour of collection. Live mites were weighed on a set of electronic scales (Kern ALJ 160-4NM) together, and an average taken, as a single mite was too small to register on the scales $(n=$ $12,0.42 \mathrm{mg})$.

\subsection{Visual and vibrational measurements}

Mites were placed either onto (i) a plastic Petri-dish $(50 \mathrm{~mm}$ $\times 10 \mathrm{~mm}$ ), (ii) a "British Standard" sized frame of fully built, but empty comb or (iii) a piece of loaded brood-comb, removed from a hive using a scalpel (two samples were used: $1-3.5 \times 2 \mathrm{~cm}$ diameter, containing 6 empty cells and 25 capped brood cells, and $2-4 \times 2 \mathrm{~cm}$ diameter, containing 17 empty cells and 20 capped brood cells). An ultra-sensitive accelerometer (4507 B 002, Brüel and Kjær, $1000 \mathrm{mV} / \mathrm{g}$ ) was used to collect the vibrational data, glued to the Petri-dish or secured centrally to the frame of comb by pushing it into the comb and adding a small amount of molten wax to hold it in place. Brood-comb samples were placed on the Petridish with accelerometer attached. The activity of the mites on each substrate was filmed and the vibrational data synchronously recorded with a camera (Sony 4K FDR-AX100E handycam, China). Each filming session lasted from 10 to 20 minutes, for all three substrates. Mites were placed on each substrate in randomly selected groups (ranging from 1 individual to 19 individuals), dependent on the number of mites collected that day. Overall, the number of videos recorded 
for each are as follows: i) Petri-dish $(n=10)$, ii) honeycomb $(n=5)$, iii) brood-comb $(n=2)$. The full technical details for this set-up and all further experimental design can be found in supplementary material S1.

The recordings were then examined to identify behaviours of interest. Several behaviours were identified that produced a detectable vibrational trace (supplementary Fig. S2, S3, S4 and S5), but we chose to focus on those that were deemed the most promising for yielding exciting science and for further analysis. The pulse that is the main focus of this study is a vibrational trace that is produced by a novel behaviour, which we term 'jolting'. Attempts to capture acoustic sound from this behaviour with both a standard microphone (Tascam DR-05X, USA) and ultrasonic bat detector (Magenta Bat 5, Tutbury, UK) were unsuccessful, indicating that jolting pulses produce vibratory signals only.

For all recordings (265 minutes of footage in total) where more than one mite was in the field of view, each individual mite was tracked to record the corresponding number of jolting pulses produced, in order to obtain the percentages of mites that produced the behaviour of interest.

For in-depth analysis of jolting pulse features, three videos comprising of a (different) jolting mite individual were chosen, one for each of the three substrates. Focus was placed upon these individuals as they produced a high number of the pulses of interest in succession with visible and hearable accelerometer traces.

\subsection{Comparing Varroa vibrations with honeybee vibrations}

The strongest Varroa jolting accelerometer waveform on each substrate was compared with (i) mite walking vibrations, (ii) the vibrations of an individual honeybee and (iii) those originating from a full honeybee colony. The strongest jolting pulse was initially chosen to demonstrate the current best-case scenario for discriminating between the vibrational trace of jolting and those of honeybee and Varroa behaviours. The magnitude of acceleration of every jolting pulse in the three collections was then also compared to the vibrational traces of honeybees and Varroa behaviours to provide an overall comparison.

'Low' and 'high' signal recordings of the honeybee colony (depending on the comb mass density load) were collected for the full colony comparison. The entire colony is distributed on ten frames, resulting in typically 1000 to 2000 honeybees per frame, and we expect that around $10 \%$ of these individuals contributed to the measured signal.

An individual honeybee vibratory signal (a 'whooping' signal (Ramsey et al. 2017)), was also extracted from this data, for the individual honeybee comparison.

The brood-comb substrate data was chosen for visual comparison to the colony and individual honeybee vibrations as the brood-comb set-up is (i) the closest to the mites' natu- ral environment, and (ii) is the best match of substrate types onto which accelerometers were attached. This allowed us to explore the possibility of detecting mites amidst the continuous honeybee signal.

\subsection{Estimating the power required for Varroa jolting pulse production}

The power output of the jolting pulse (i.e. the muscular power required by the animal to produce the vibration (Michelsen et al. 1982)) was estimated by an experiment aiming at replicating the vibrational trace of the Varroa jolting signal. Glass beads ( $400 \mu \mathrm{m}$ in diameter) were dropped from a height of $1 \mathrm{~cm}$ onto the Petri-dish, both directly above and slightly offset the accelerometer, to replicate the differing positions of mites when jolting. Fifteen repeats were taken for each position.

The bead velocity was measured by estimating the distance travelled by the bead frame-by-frame as it was dropped, using a video captured at 240 frames-per-second (FPS). The average weight of a collection of glass beads was taken as a single bead was too light to be detected by the scales (8028-series professional digital jewellery scale, China) (0.07 mg).

\subsection{Signal analysis}

All signal analysis was conducted through Matlab (Refworks 2019a) using code written specifically for this study at Nottingham Trent University. The vibrational trace of the jolting behaviour was analysed directly from the audio track of the video footage. The synchronicity between the vibrational trace and the movement of the Varroa mite was demonstrated through the creation of a separate movie (one for each of the substrates investigated). Each jolting pulse vibrational trace was extracted from the accelerometer data and further analysed using their waveforms and spectrograms. All the pulses in each substrate collection were carefully synchronised using the cross-correlation product so that comparisons could be made within the three collections. Two methods of alignment were used, dependent on the type of analysis that was to be performed. Varroa walking pulse vibrational traces were also extracted from the three recordings for comparison against the jolting pulses on each respective substrate.

\subsection{Signal to noise ratio analysis/successful detection of jolting pulses}

To establish how detectable the jolting pulse is, by accelerometer evidence only, the signal-to-noise-ratio (SNR) value was first estimated for each substrate and each jolting pulse: Petri-dish, empty honeycomb and brood-comb. Critical listening of the accelerometer recording of the signals was then undertaken, separately. Playback speeds for each substrate were reduced accordingly, to best demonstrate the pulses audibly (Petri-dish reduced by a factor ten, honeycomb and brood-comb reduced by a factor 2). The percentage of audi- 
ble signals was finally calculated to pinpoint the (independently calculated) SNR threshold value, indicating where the method begins to detect jolting signals.

\subsection{Accelerometer and Petri-dish resonance analysis}

To measure the inherent resonance of the accelerometer that is used in our work, a full frequency sweep $(0-24000 \mathrm{~Hz})$ was conducted, using the same equipment as for the rest of the study. The amplitude of the accelerometer recordings, taken at three signal magnitudes, was compared by plotting the ratio between the signal sizes. The Petri-dish with attached accelerometer was also measured with a full frequency sweep $(0-24000 \mathrm{~Hz})$ to establish the frequency response and to then compare it to that of the accelerometer alone. The responses of the Petri-dish and accelerometer were then visualised together.

\section{Results}

\subsection{The behaviour of interest: the jolting pulse}

The vibration that is the focus of this study, here termed 'jolting pulse', chosen because of the corresponding abrupt movement of the body, is extensively showcased and analysed. We describe this behaviour as a rapid pulsing of the body either in a left or right direction, before returning to the original central resting position (supplementary video S6). When producing a jolting pulse and viewed from a side-on position, the Varroa legs can also occasionally be observed to flex, moving the body down and then back up again off the substrate (supplementary video S7). During all video observations, mites were only observed to jolt when stationary.

Mite jolting behaviour was recorded and analysed on three substrates (empty honeycomb, brood-comb and Petridish). Overall, 55 mites were inspected for general jolting patterns. $46 \%$ of these were never observed to jolt. The remaining 54\% jolted at some stage during the video footage, categorised as either jolting without an obvious trend $(42 \%)$, or jolting with an obvious trend (13\%) (supplementary Fig. S8). We define 'obvious trend' as a mite repeatedly jolting in quick succession, with 10 seconds or less between consecutive jolts for more than one minute following commencement of the behaviour.

A substantial number of jolting vibrational pulses were measured on Petri-dish $(n=250)$ and on brood comb $(n=$ $189)$, with a smaller sample size for honeycomb $(n=28)$. Other vibrational traces were also detected when mites produced recognisable motions such as walking (supplementary video S2). All observed behaviours except one (defecation) produced measurable accelerometer traces (supplementary videos $\mathrm{S} 3$, S4 and $\mathrm{S} 5$ ).

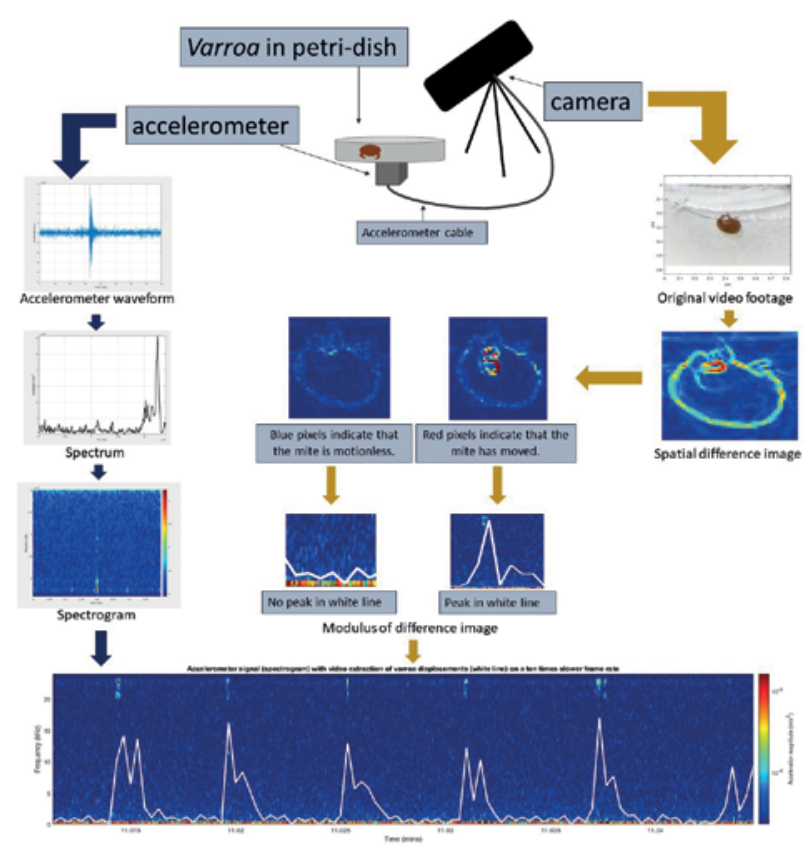

Fig. 1. A diagram to demonstrate the process of analysing the jolting pulse data. All three mites on each substrate underwent the same video analysis. The mite is placed on the substrate with the accelerometer attached and is filmed with the camera, producing a movie that has synchronous audio and video. The lefthand side of this diagram demonstrates how the vibrational data are processed. The raw accelerometer waveform of the movie is transformed into a spectrum, which shows the frequency $(\mathrm{Hz})$ ( $x$ axis) and magnitude of acceleration $\left(\mathrm{m} / \mathrm{s}^{2}\right)$ (y axis) of the vibrational data. A collection of such spectra are then stacked into a spectrogram, which shows time (seconds) (x axis), frequency $(\mathrm{Hz})$ (y axis) and magnitude of acceleration (logarithmic to the base 10) (pixel intensity). Dark red shows the highest magnitude and dark blue shows one $70^{\text {th }}$ of this maximum. The maximum acceleration magnitude is forced to be that of the Varroa jolting pulses for better viewing, resulting in clipping of the irrelevant data at the lowest frequencies. The accelerometer recording for the entire original movie is transformed into a spectrogram, running with respect to time. The bottom panel shows a two-seconds long section of this movie. The right-hand side of the diagram demonstrates how the visual data are processed. The original video data that is collected is further cropped to better focus on the mite for the purpose of simple edge detection (by means of the spatial gradient of the pixel intensity). The modulus (absolute value) of the difference image shows the changes in pixel intensity in two consecutive frames as seen in the spatial difference image. In the modulus of the difference image, the mite is mostly dark blue when motionless, but exhibits edges that flash red when moving abruptly. The sum of the pixel intensities in this panel are then displayed as the white line that is superimposed on the spectrogram data of the bottom panel. This demonstrates the remarkable synchronicity between video-detected mite displacement and accelerometer trace. This processing is used to create the supplementary videos S9, S10 and S11. 


\subsection{Relating the Varroa vibrational pulse to the video recording of jolting}

The absolute timing of each jolt accelerometer trace was assessed within the sampling rate used for that signal, i.e. $48000 \mathrm{~Hz}$. The vibrational trace is systematically seen (Fig. 1) to be produced within the time window of the videodetected mite jolting displacement, which is assessed within an interval of two successive video frames i.e. $20 \mathrm{~ms}$. Despite this large discrepancy in the sampling rates of the two independent assessments of the jolting, our analysis demonstrates (Fig. 1) that the observed vibrational trace and the jolting behaviour of the mite are consistently synchronous phenomena to within $20 \mathrm{~ms}$ (Fig. 1, supplementary videos S9, S10, S11). Figure 1 also demonstrates that the visually assessed time duration of the jolting (the width of the white peaks) is substantially longer to that assessed from the vibration (the spectrogram traces).

\subsection{Effect of substrate}

The Varroa jolting vibrational trace that exhibited the highest magnitude was extracted from each substrate-specific collection of measurements (Fig. 2). This trace varies substantially, in shape mostly, and modestly in time duration, dependent on the substrate on which the mite resides. The substrate-specific features observed in the loudest pulses can also be seen in most of the jolting vibrational traces. On the Petri-dish, the signal is mostly found at the highest measured frequencies (19 to $23 \mathrm{kHz}$ ), with the strongest signal contribution found between 22 and $23 \mathrm{kHz}$ (Fig. 2, supplementary video S12, supplementary Fig. S13). On the brood-comb, the opposite situation occurs, with the signal of highest magnitude found at the lowest frequencies between 1 and $1.5 \mathrm{kHz}$ (Fig. 2, supplementary video S14, supplementary Fig. S15). In the honeycomb the signal is found over a much broader bandwidth between 0.5 and $10 \mathrm{kHz}$ (Fig. 2, supplementary video S16, supplementary Fig. S17, supplementary Fig. S18). As the pulses in Fig. 2 are exceptionally strong, the Varroa jolting pulses also stimulate a collection of frequency bands rarely visible on the full collection of spectra.

The maximum acceleration values of the strongest jolts are similar between the honeycomb $\left(0.0032 \mathrm{~m} / \mathrm{s}^{2}\right)$ and brood-comb $\left(0.0035 \mathrm{~m} / \mathrm{s}^{2}\right)$, with a stronger acceleration for the loudest Petri-dish pulse $\left(0.005 \mathrm{~m} / \mathrm{s}^{2}\right)$ (Fig. 2). In spite of this, the trace on the honeycomb spectrogram is noticeably fainter (typically reaching the yellow colour, i.e. approximately 5 times weaker than the signal seen otherwise in the
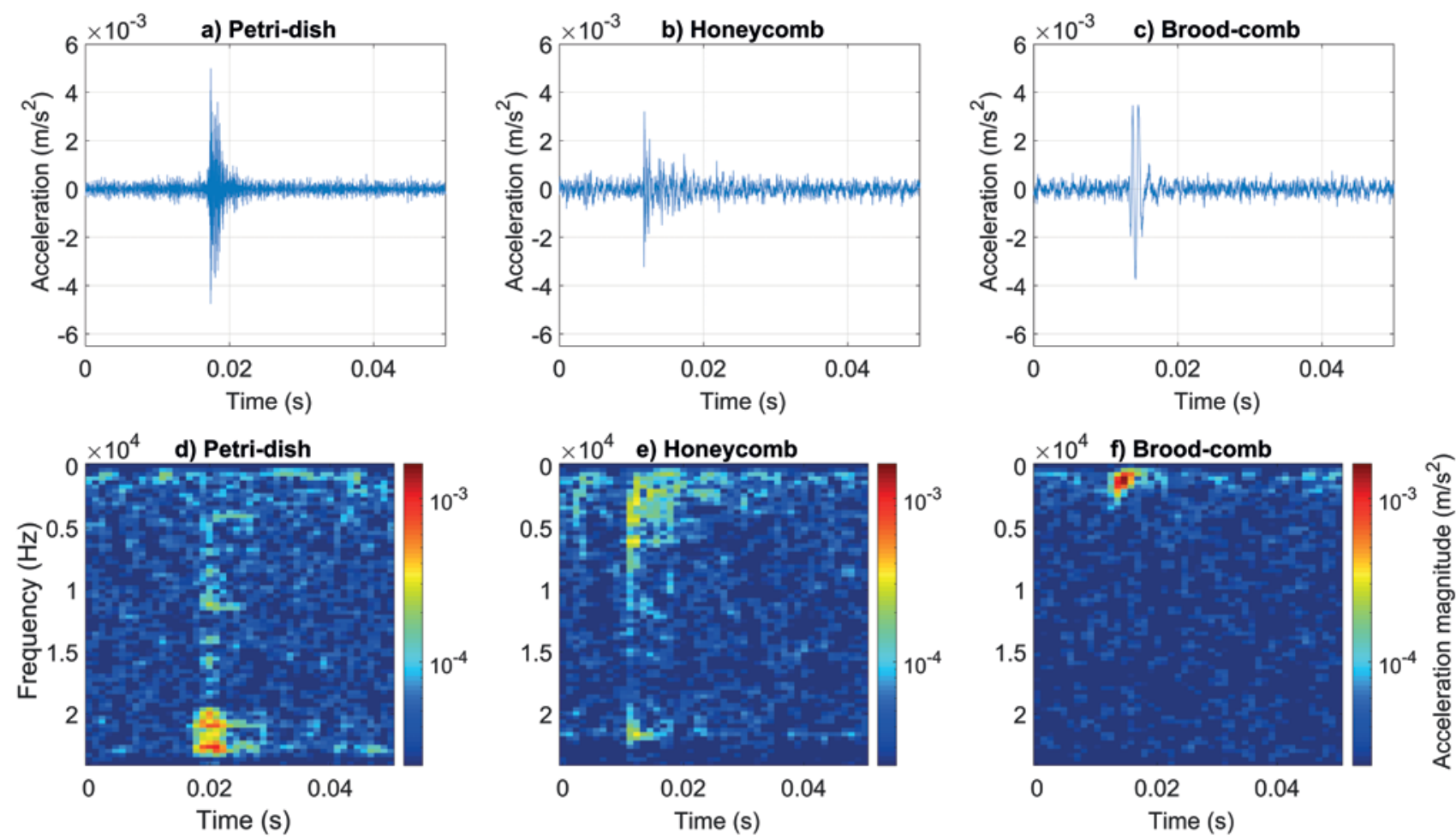

Fig. 2. A series of accelerometer waveforms (top) and spectrograms (bottom) allowing the comparison of the vibration originating from the strongest Varroa jolt, detected within three separate substrates (Petri-dish (a and d), empty honeycomb (b and e), and broodcomb ( $c$ and f). The accelerometer waveform has been high-pass filtered ( $500 \mathrm{~Hz}$ cut-off for all substrates) to remove irrelevant background vibrations that otherwise dominate the waveform. In the spectrogram, acceleration magnitude is logarithmic (to the base 10), where the highest magnitude is dark red $\left(1.6 \times 10^{-3} \mathrm{~m} / \mathrm{s}^{2}\right)$ and the lowest magnitude dark blue (and forced to $1 / 70$ of the maximum). Panels found in the top, and the bottom, are scaled identically for ease of comparison. 
deep red band), due to the signal being spread over a remarkably broad band.

All jolting pulses have an exceptionally short time duration, regardless of the substrate. The jolting pulse appears as a single, rapid pulse that takes between $0.05 \mathrm{~ms}$ and $0.09 \mathrm{~ms}$ to grow, exponentially, from the start of the signal to maximum acceleration on the honeycomb and Petri-dish substrates, respectively. On the brood-comb, the growing wave front appears gaussian, with a $1 \mathrm{~ms}$ typical time duration (supplementary Fig. S19).

Jolting waveforms registered in both honeycomb and Petri-dish can be described as damped sine waves, with a typical decay constant of $0.1 \mathrm{~ms}$ and $1.2 \mathrm{~ms}$ respectively for the strongest pulses (Supplementary Fig. S19). To assess the full pulse collections, average decay constants $(\lambda)$ were calculated after visual fitting on the clearest individual waveforms to quantitate the variation in the population of pulses (Petri-dish $(n=20) \lambda=1.94 \mathrm{~ms}$ (mean), $1.03 \mathrm{~ms}$ (s.d.), honeycomb $(n=5) \lambda=0.5 \mathrm{~ms}$ (mean), $0.37 \mathrm{~ms}$ (s.d.)).

Separate, repeatable features observed in the pulses detected within the brood-comb substrate prompted us to select the 40 traces with the highest magnitudes, and to average them to showcase the mean accelerometer waveform characteristics. The envelope of the corresponding broodcomb pulse is very well described by a gaussian function (supplementary Fig. S19), with an average full width at halfmaximum of $1.33 \mathrm{~ms}$. It is worth noting that there is also a visible, but negligible, exponential decay of the signal.

\subsection{Within substrate comparison}

The strongest jolting pulses are not necessarily representative of the whole collection of measurements as deviations from pulse to pulse can take place. Amongst the three substrates, jolting pulses detected within the brood-comb exhibit the highest repeatability (Fig. 3 for the waveforms, supplementary Fig. S15 for the corresponding power spectra). All pulses are 4 to $10 \mathrm{~ms}$ long, with three to five main lobes probably originating from one of the substrate's resonant mode. A few Varroa pulses (3\%, based upon visual examination of the spectrograms and waveforms) exhibit a double peak (e.g. the tenth pulse on Fig. 3), which, upon inspection of the video, appears to be produced by a matching rapid, repeated motion of the Varroa body (supplementary video S20). When slowed down appropriately, jolting pulses in this video can be heard as 'knocking' noises, with the double trace pulses producing two separate knocks.

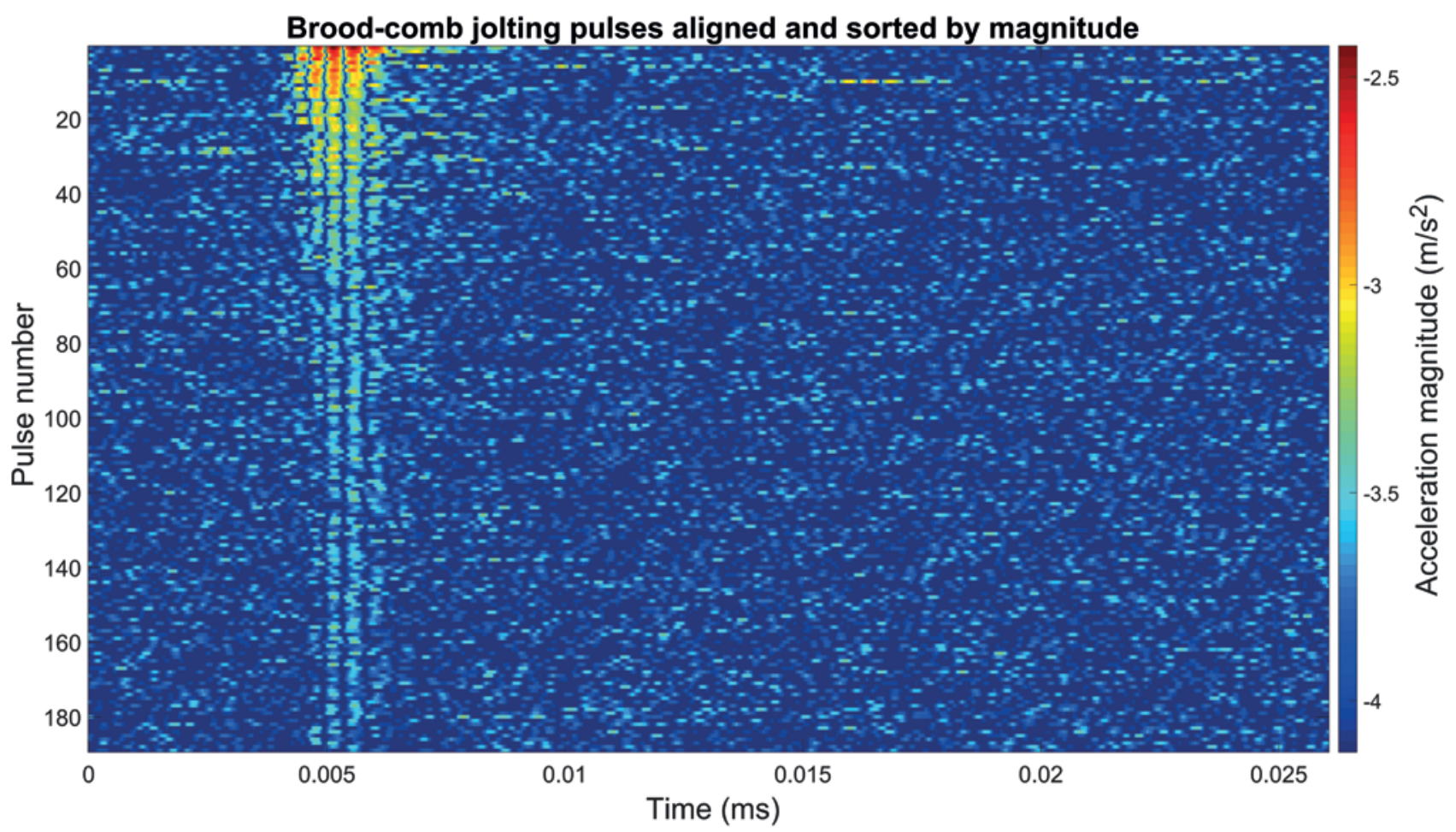

Fig. 3. The collection of all 189 jolting pulses detected in the brood-comb after under-going cross-correlation alignment with the reference pulse (pulse number 1 in this instance), and further sorted by magnitude, in decreasing order. The similarity between pulses observed in this figure is remarkable. The polarity of acceleration has been forced to be positive to allow its magnitude to be shown on a logarithmic (log base 10) scale with dark red showing as the highest magnitude $\left(3.4 \times 10^{-3} \mathrm{~m} / \mathrm{s}^{2}\right)$ and dark blue showing as the minimum magnitude (1/50 of the maximum). 
In the case of the Petri-dish substrate, most of the visible traces in spectrogram format are short, single peaks mostly found at the ultra-high frequency range, again with a few instances exhibiting more than one peak in quick succession (supplementary video S12). However, careful inspection of the original Petri-dish Varroa jolting video does not reveal behaviour changes that could be linked to the production of multiple peaks (supplementary video S9).

Of the 40 strongest jolting pulses on the Petri-dish 25\% $(n=10)$ of the spectra also exhibit lower frequency bands, additional to the ultra-high frequency band (supplementary Fig. S21). These are found around 10.6 to $11.3 \mathrm{kHz}, 13.4$ to $14.1 \mathrm{kHz}$, and 15.1 to $15.8 \mathrm{kHz}$. Of this $25 \%$, seven are found in the top ten strongest of the whole sample.

Varroa jolting pulses registered in the empty honeycomb substrate also exhibit variation. Some of the visible honeycomb pulses comprise of a 'train' of several pulses in quick succession. An exceptional instance of this can be seen (supplementary Fig. S22) with six clear consecutive traces within $10 \mathrm{~ms}$ of recording. In this case, when observing the mite on the video footage, the expected behaviour is not seen. Instead, the mite jolts its body to the right and then in a forward motion, rather than back to the left (sup- plementary video S23). This movement has not been seen in other pulses that produce multiple peaks, but still yields vibrational pulses with spectral features identical to those of a jolting behaviour.

\subsection{Comparison of jolting pulse with other vibrational traces}

Here, the Varroa jolting accelerometer waveforms are compared to those originating from (i) the mite walking and (ii) honeybees. We first compare the strongest jolting pulse with the signal captured from a full honeybee colony, using the brood-comb data for the comparison (Fig. 4). The strongest Varroa pulse is compared with a single honeybee 'whooping' signal, and the overall signal originating from the colony during two phases: low accelerometer signal (frame is heavily loaded with brood and/or honey, therefore attenuating vibrational signals) and high accelerometer signal (frame is mostly empty, therefore less attenuation occurs). Despite the large difference in mass and size between an adult honeybee individual $(115 \pm 7 \mathrm{mg}$ (Schmolz et al. 2005), $15 \mathrm{~mm})$ and a Varroa mite $(0.42 \mathrm{mg}, 1 \mathrm{~mm})$, it is most surprising to find that the acceleration magnitude of the strongest jolting pulse is comparable to that of the colony

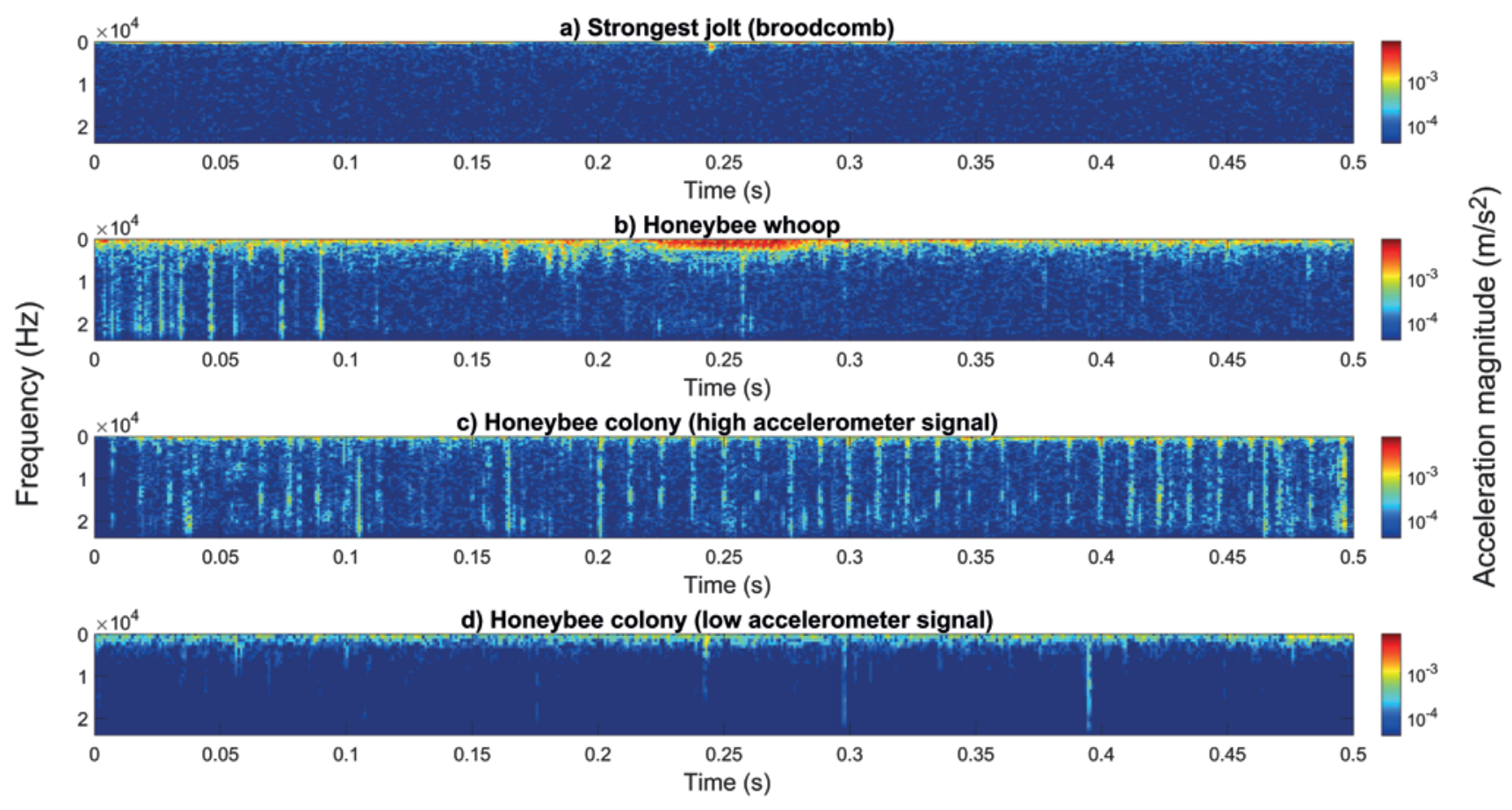

Fig. 4. A series of 0.5 s long spectrograms allowing the comparison of the vibration originating from the strongest Varroa jolt on broodcomb (panel a), a honeybee whooping signal that comprises of a single pulse (panel b), and the whole honeybee colony in both low signal (panel c) and high signal periods (panel d). Variation in signal strength between 'c' and 'd' occurs mostly as a result of changes in comb mass density throughout the 21 -day period of the brood-cycle. In this figure, the magnitude of the acceleration is logarithmic (to the base 10), where the highest magnitude is dark red $\left(7.5 \times 10^{-3} \mathrm{~m} / \mathrm{s}^{2}\right)$ and the lowest magnitude dark blue (and forced to $1 / 200$ of the maximum). All four panels are scaled identically for ease of comparison. In the top spectrogram, the regular background vibration inherent to the room was calculated and subtracted. 

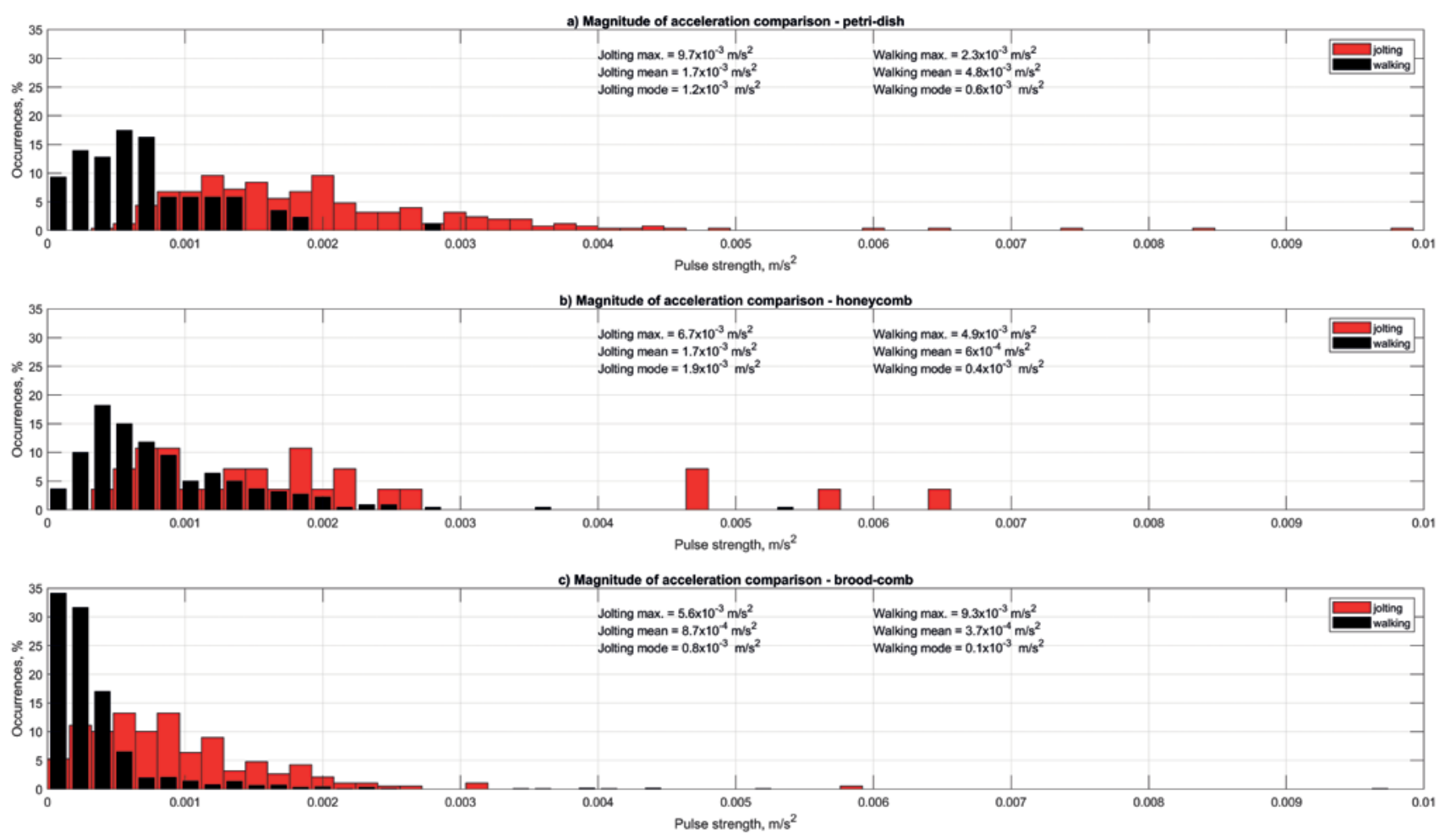

Fig. 5. A series of histograms demonstrating the strength of Varroa jolting against the walking pulses on each substrate. In all three panels the vertical axis is normalised to showcase the distribution probability (Petri-dish jolting/walking pulses $n=250 / 89$, honeycomb jolting/walking pulses $n=28 / 219$, brood-comb jolting/walking pulses $n=189 / 1272$ ). Histogram bin-width is identical for all plots - but the displayed black walking bars have been narrowed so that the red jolting bars, displayed behind, can be viewed.

during a period of low signal (jolting pulse $=9.4 \times 10^{-3} \mathrm{~m} / \mathrm{s}^{2}$, low signal colony $\left.=1.9 \times 10^{-3} \mathrm{~m} / \mathrm{s}^{2}\right)$. The jolting pulse is also only five times smaller than (i) an individual pulsed bee signal and (ii) the entire colony when accelerometer signal is high (Fig. 4, supplementary Fig. S24) (jolting pulse $=$ $9.4 \times 10^{-3} \mathrm{~m} / \mathrm{s}^{2}$, whooping signal $=4.5 \times 10^{-2} \mathrm{~m} / \mathrm{s}^{2}$, high signal colony $=4.5 \times 10^{-2} \mathrm{~m} / \mathrm{s}^{2}$ ). The quoted magnitudes of acceleration represent the integral of all acceleration frequency components for the maximum vertical point in time in each panel (supplementary Fig. S24).

The strongest jolting pulse captured on the empty honeycomb also demonstrates the same order of magnitude as the vibrations of the honeybee colony (loudest honeycomb jolting $=9.2 \times 10^{-3} \mathrm{~m} / \mathrm{s}^{2}$ (supplementary Fig. S25), registered from within a normally loaded frame.

The jolting vibrations are then compared to walking mite vibrations, registered on all three substrates (supplementary Fig. S26). On each of the three substrates, the maximum magnitude of acceleration is calculated for every jolting pulse, and a large collection of walking pulses, for comparison. Although for the case of the brood-comb, in rare instances, walking vibrations can be stronger than jolting pulses, the average jolting pulse strength is systematically higher than that of the walking pulses on all substrates (Fig. 5).
The spectra of the walking and jolting pulses are then compared to each other to explore whether they could be discriminated (supplementary Fig. S27). The vibrational pulses originating from a Varroa walking on all three substrates are produced regularly at a frequency ranging from 0.5 to $3 \mathrm{kHz}$. On the Petri-dish, the $0.5 \mathrm{kHz}$ peak may be a resonance of the Petri-dish, as it is the only frequency clearly observed as a result of stimulation to this substrate (supplementary Fig. S28). On the brood-comb, the vibrations produced by both behaviours share the same frequency band, whereas the Petri-dish and honeycomb demonstrate high discrimination. Both the jolting and walking pulses on honeycomb stimulate the ultra-high frequency bandwidth that seems to originate from a resonant mode inherent to the accelerometer itself (supplementary Fig. S29).

\subsection{Estimation of pulse power output}

To estimate the power output provided by a mite delivering a jolting pulse, a well-controlled vibration was produced artificially, yielding a vibrational trace similar to that of a Varroa.

The kinetic energy of the bead hitting the Petri-dish (see 'Methods') was estimated to be $17.5 \mathrm{~nJ}$, which, when delivered within $0.54 \mathrm{~ms}$ (the time duration of a jolting pulse to 

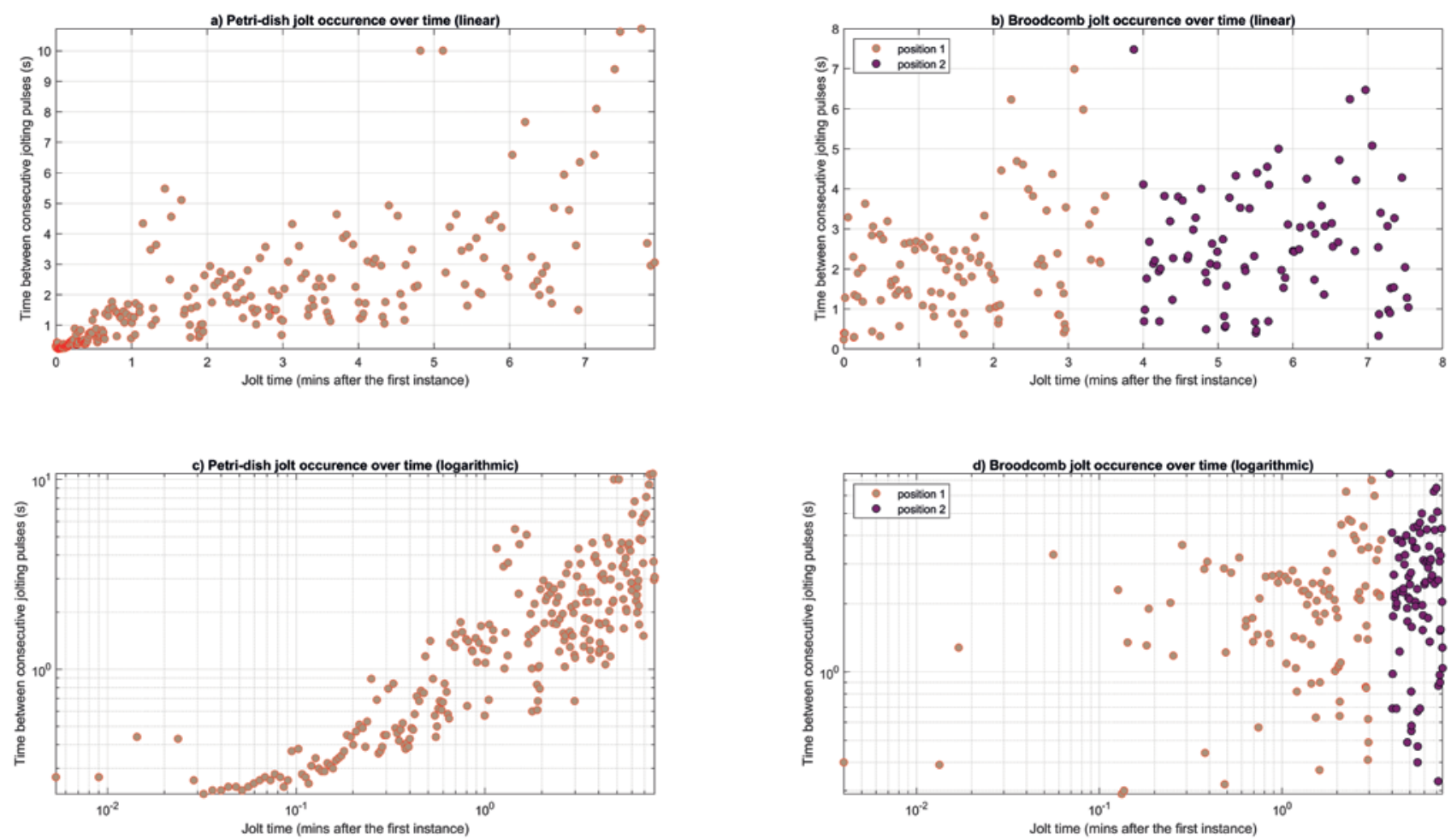

Fig. 6. Jolt occurrence time intervals with respect to time. The data for the mite jolting on Petri-dish (left column) and on brood-comb (right column) are shown here. Jolt occurrence time intervals are showcased in both linear (a, b) and logarithmic (c, d) scales. A change in the colour of the data points is indicative of the mite moving to a new position on the substrate.

go from 0 to maximum on the Petri-dish (supplementary Fig. S19) results in a power of approximately $32 \mu \mathrm{W}$. As the mite vibrational pulse is forty times weaker than the artificially produced vibration, this means that the animal must typically deliver approximately forty times less power, i.e. $810 \mathrm{nW}$ in each jolting pulse.

\subsection{The detectability of jolting pulses using accelerometer sensors}

Not all video-evidenced jolting pulses produced a detectable vibrational trace. This must be due to the animal delivering vibrations with varying strength. The fraction of detected pulses decreases with a reduction in the accelerometer's sensitivity, and no jolting vibration was ever detected when using a ten-times less sensitive crystal (4507, Brüel \& Kjær, $100 \mathrm{mV} / \mathrm{g}$ ).

In order to assess the detectability of jolting vibrational pulses on each substrate, the signal to noise ratio (SNR) was estimated for each vibrational trace, and we also independently noted the percentage of pulses that could be picked up by critical listening, to best perceive the collection of jolting pulses. On the brood-comb, $25 \%$ of pulses were hearable, corresponding to a SNR boundary of 0.7475 . Petri-dish and honeycomb exhibited a higher percentage of audible pulses,
$47 \%($ SNR boundary $=0.0142)$ and $68 \%($ SNR boundary $=$ $0.2265)$, respectively.

\subsection{Periodicity of the behaviour}

We now consider all the mite individuals observed for jolting behaviour (supplementary Fig. S8), and describe the periodicity and pattern of the jolting. Mites produced this behaviour for varying lengths of time (from as little as one single jolt up to almost 50 minutes of regular jolting behaviour), often with a few location changes between where jolting was seen to temporarily cease.

Of the mites that were observed jolting $(54.5 \%, n=30)$, individuals were categorised based upon the greatest length of time during which the behaviour was regularly produced. We call this a 'bout' of jolting, defined as a period where a mite remains in the same position and jolts with 10 seconds or less between consecutive pulses. If more than 10 seconds elapsed, or mite re-positioning occurred, then the subsequent jolt that followed this was considered to be the start of a new bout. $77 \%$ of these mites jolted for periods of less than one minute (supplementary Fig. S8, panel 'b'). Mites producing a jolting bout that lasted more than one minute $(13 \%)$ were then further scrutinised to identify an overall behaviour pattern during their time spent on the substrate. 
The overall duration of jolting varied between individuals, with the longest time-period observed as 49 minutes and 50 seconds. The Petri-dish and brood-comb mites pulsed for approximately 8 minutes each (Fig. 6). The periodicity of the jolting of the mite individual residing on the empty honeycomb is also shown alongside the data for other mites (supplementary Fig. S30) with broadly varying overall pulsing time durations demonstrated. In $50 \%$ of cases in this category, mites repositioned during jolting. This was either a minor displacement, where the mite simply stopped pulsing to turn and face another direction, or a larger motion, where it stopped then walked to another area on the substrate before immediately continuing with the behaviour.

For some mites, there appears to be a trend in their pulsing activity, but modest sample size prevents us from drawing interpretive comments. Some individuals jolt rapidly from commencement of the behaviour, with very short time intervals, around 0.5 seconds, between consecutive jolting pulses. As time increases, the temporal density of pulse occurrences decreases (see Fig. 6, panels ' $a$ ' and 'c', see also supplementary Fig. S3, panels ' $a$ ' to 'd'). Other individuals produce jolting pulses at more regular intervals regardless of time, with a similar rest period between consecutive pulses (Fig. 6, panels 'b' and 'd', supplementary Fig. S30, panels ' $e$ ' to ' $h$ '). Where repositioning occurs, identified by a change in colour on the figures (Fig. 6, supplementary Fig. S30) the trends here described appear to continue regardless of the interruption due to movement.

\section{Discussion}

In this work, we detected vibrational signals originating from individual Varroa mites with accelerometer sensors. As the jolting behaviour is very common, it comes to a great surprise that it has, until now, remained unnoticed. Only one previous study describes 'jolting movements' in Varroa (Piou et al. 2019), however this work instead characterised the reaction of mites to electrostatic substrate discharges. In our study, more than $50 \%$ of mites produced jolting behaviour at some stage during our observations, ranging from single jolts to mites that jolted hundreds of times for several minutes.

The synchronicity between video-captured mite movements and accelerometer vibrational traces provides strong evidence that the displacements of the body in left and right directions are features of the same behaviour which also yields a detectable vibration. Critical listening of the audio of the slowed video data also further demonstrates a robust synchronicity where the jolting pulses can be heard as 'knocking', 'clapping' or 'clinking' noises, with a timbre clearly modulated by the nature of the substrate in which the vibration is detected.

On all three substrates investigated, double or multiple peak vibrations were occasionally found in rapid succes- sion. On brood-comb and honeycomb, two rapid, successive body displacements can be seen alongside a double audible 'knock' or 'clap' consistent with the multiple peak vibratory trace seen. For Petri-dish, although visible differences in the jolting vibrational traces are seen (some have single peaks, some multiple peaks), there are no visual or audible differences in the production of both types of pulse when viewing the displacement of the mite. Perhaps the double motion of the body observed on the brood-comb and honeycomb is a result of the uneven comb surface interfering with the flow of the displacement, whereas the Petri-dish is homogenous and flat.

The mechanism behind jolting vibration production can only be speculated presently. The visible motion of the body is approximately $20 \mathrm{~ms}$, the vibrational trace itself is only 0.3 to $1 \mathrm{~ms}$ long, followed by a decay of 1 to $4 \mathrm{~ms}$ that is likely to result from the resonance of the substrate. This could be indicative of a 'spring' or 'click' mechanism, which is documented in a variety of species that produce ultra-short and ultra-fast movements (Gronenberg 1996; Patek et al. 2011). The relatively slow visual jolting would correspond to the loading of the 'spring', whilst its ultrafast release would cause the remarkably short accelerometer pulse.

\subsection{Substrate dependent jolting pulse features}

Dependent on the substrate on which it is transmitted, the jolting pulse vibrational trace varies substantially, as would be expected from any vibrational signal captured on a variety of media. In the honeycomb, in particular, there is a remarkably broad band trace that could be described as resulting from a Dirac Delta function (note, also, that for similar accelerometer traces, the broader the spectral band is, the weaker the signal magnitude registered on the spectrum, necessarily, as seen in Fig. 2). This corresponds to an ultra-fast burst of energy delivered to the substrate (Reeping \& Reid 2016). A jolting mite delivers a short burst of vibrational energy into the material on which it stands and the shorter this pulse is, the broader the environmental information gathered in the spectrum we register. The pulse growth appears to be the only part of the vibration highly specific to the mite. The oscillations following it, which contribute to the overall time duration of the pulse, must result from the vibrational energy gradually dissipating in the substrate.

The platform on which a vibration is transmitted produces a set of resonance signals that are characteristic of the substrate (Otten et al. 2001), a strong feature that we see in our results. Stronger Petri-dish and honeycomb jolting pulses stimulated an increased number of frequency bandwidths than lower intensity ones, an indication that more resonance information is produced (Otten et al. 2001). Jolting pulses in general stimulate more frequencies than walking pulses, which have a lower frequency bandwidth of 0.5 to $3 \mathrm{kHz}$, irrespective of the substrate they were delivered on. It is perhaps the result of the natural responding frequencies of the 
substrate that cause brood-comb jolting and walking pulses to share a similar bandwidth.

The main resonance of the accelerometer used $(17.9 \mathrm{kHz})$ was coincidentally never stimulated by mite activities. Deviation from signal strength linearity at the moderate resonances of the crystal $(11,15$ and $22 \mathrm{kHz}$ ) was less than $10 \%$ (supplementary Fig. S29), further indicating that the frequency components of the jolting pulses were a genuine result of mite stimulation.

\subsection{Successful accelerometer detection of mite signals}

Accelerometers have been used successfully by our research group to detect and characterise specific honey bee signals (Bencsik et al. 2015; Ramsey et al. 2018; Ramsey et al. 2020), and we have now demonstrated that the same sensors can also detect the vibrations of a much smaller and lighter organism, V.destructor, on three different substrates. Varroa jolting signal capture is particularly successful on the Petri-dish and honeycomb substrates, but still demonstrates an efficacy of $25 \%$ on the denser brood-comb. This is still remarkably good, considering that capped brood-comb is known to be poorer at transmitting vibrational signals in comparison to open, empty cells (Bencsik et al. 2015).

\subsection{Application of jolting pulse detection in beehives}

Our results suggest that it might be possible to continuously, non-invasively detect live Varroa mites within fully populated hives using accelerometers. We have demonstrated that this signal is highly repeatable, with an identifiable shape, spectrum and time duration, including for Varroa residing on brood-comb, which is the most similar to a real hive substrate.

The average strength of the jolting pulse is well above what would be expected for an animal of such a small size. The vibrational strength of the honeybee colony recording, in which hundreds of bees contribute to the measurement during a period of high signal, is only one order of magnitude higher than that of a single mite jolting pulse. We can see in our data that when there are lulls in bee buzzing, jolting pulses exceed the maximum acceleration of the bee signal, providing a good opportunity for them to be detected. The high signal data strength is, even more surprisingly, comparable to that of the jolting, although there is a limitation to this result: The honeybee vibrations were emanating from an entire hive's frame, which during the low signal period was loaded with brood/honey, whilst the jolting pulse, in comparison, was delivered onto a much smaller section of brood-comb. This perhaps led to the comparable signal strength seen in the low signal data when compared to the jolting pulse. Nonetheless, we have shown that honeycomb pulses are comparable in strength to brood-comb pulses, and these were recorded on a British standard sized frame which, although devoid of contents, was still fully built in terms of the wax cells, i.e close to the expected substrate in the hive. This is especially true for the high signal hive data, as the frame will be closer to a state of emptiness due to a lack of brood e.g. when eggs have just been laid, after a swarm and during winter (Bencsik et al. 2015). Success in detecting Varroa vibrational signals in the hive is therefore expected to vary throughout the year in response to the everchanging periods of high and low signal that occur due to the broodcycle and therefore shifts in frame load (Bencsik et al. 2015).

It is most likely that mites would be successfully detected when within the brood cells, as population modelling predicts that $65 \%$ of mites in a honeybee colony will be within the sealed cells at any time (Martin 1998). Varroa have phases where they come into direct contact with the comb in the reproductive phase (Donzé and Guerin 1994), so walking will take place and it is possible that jolting may also occur. We know from our experience that bees fill the cells surrounding the accelerometer with their normal content, including brood. This increases the chances of a mite inhabiting the vicinity of the sensor for many days, improving the chance of its detection. Establishing the function of the jolting pulse may improve mite detection, as it is currently unknown whether it is produced in the brood cells. If we establish when and where the behaviour is likely to occur, we can focus on more specific search times and locations for its detection. In previous studies, we have placed two accelerometers in a central position on the frame, equidistant from one another and the frame edge. This has worked well for registering frequently occurring honeybee signals (Ramsey et al. 2018) but may not be optimum for mite signal capture, particularly as detection success is likely to be affected by the distance between the mite and accelerometer. In our work so far, mites were always positioned within three to four $\mathrm{cm}$ of the sensor, but Varroa could reside everywhere in a real hive. It is likely that an array of accelerometers will be needed for this exploration.

The work discussed here therefore provides a pioneering step towards the detection of mites in a real hive using accelerometers. The features that we have extracted and described can be used to inform a search tool for jolting and walking pulses within a full-sized hive. We can now utilise this breakthrough as the foundation for future endeavours, such as investigating whether the mite vibrational pulses can be detected when originating from the capped brood cells.

Is the jolting pulse functional? - A better understanding of why the jolting pulse is transmitted by the mite may provide additional information on where the behaviour will occur, which may then improve Varroa vibration detection. Some of the features of jolting vibrational trace indicate that it may serve a purpose. Mites lack the necessary anatomical features for detecting airborne sound (Dillier et al. 2006), and they have no eyes (Dillier et al. 2006; Dowling 2015). It is therefore presumed that the other sensory systems guide the species' behaviour, such as the chemosensory and olfactory systems (Dillier et al. 2006; Zeigelmann et al. 2012), and 
perhaps vibratory information contributes to the successful completion of their life cycle. One feature that we see in our results is the remarkable strength of the jolting pulse. For an animal of its modest size and mass, the jolting vibration is exceptionally strong when compared to the vibrational signals of much larger honeybees. It also requires a large power of $810 \mathrm{nW}$, delivered by the mite. When comparing this to small species that produce functional vibratory signals (leaf cutter ant stridulatory distress signals: output $10-20 \mathrm{nW}$ (Markl 1967), organism size 1-10 mg (Roces \& Holldobler 1994), 3-16mm; Sehirus impressus (Canthophorus impressus) communication signal: output $30 \mathrm{nW}$, organism size $25 \mathrm{mg}, 6-7 \mathrm{~mm}$ (Michelsen et al. 1982); Omocestus viridulus grasshopper courtship signal: output $61 \mathrm{nW}$ (Michelsen et al. 1999), organism size 165-310 mg (Kriegbaum 1997), $1-2 \mathrm{~cm}$ ), we can see that Varroa, an animal of a significantly smaller size, produces jolting vibrations that are of a much higher power output. Due to the cost inherent to the high energy requirement of this signal, it would seem unlikely that the vibration we register is simply a by-product of the animals' activity.

Jolting pulses also differ dependent on the substrate on which they are produced, which, as previously discussed, is the result of the frequency response of the substrate. Some species engage in the production of probing pulses that are transmitted into a substrate so that the organism can detect the subsequent reflections and gain environmental information (Wäckers et al. 1998). Parasitoid wasps, mole rats and aye-ayes (Broad \& Quicke 2001; Kimchi et al. 2005; Sterling \& McCreless 2006) do so in order to identify hosts/ prey or gather knowledge on their surroundings. As jolting pulses vary as a result of the substrate upon which they are transmitted, but walking pulses do not, this could be indicative of a probing function.

We have also established that some mite individuals jolt hundreds of times, continuously, and for prolonged periods. This indicates a large expenditure of accumulated energy, and thereby further suggests that perhaps jolting is beneficial to the animal in some way. The probing pulses of the species mentioned earlier also follow a repetitive action, with parasitoid wasps transmitting vibrations repeatedly until a host is found (Wäckers et al. 1998), mole rats producing $198 \pm 15$ signals per metre of tunnel that they excavate (Kimchi et al. 2005), and aye-ayes tapping the material containing their prey at a consecutive rate of $97.7 \pm 19.9 \mathrm{~ms}$ over a range of a few seconds (Ramsier \& Dominy 2012). Pulse repetition for mites is therefore compliant with the idea of them serving an exploratory purpose, although it remains to be explained why some individuals remain static and jolting for prolonged periods, and why some only jolt a few times.

At this stage, we can only speculate a possible function for the novel jolting behaviour and its corresponding vibrational trace. The results of this work extensively characterise the features of the vibration, but do not explore why the behaviour may be produced. Better understanding of the pulse function will provide exciting avenues to explore, perhaps even allowing further infestation management strategies to be designed, such as non-chemical traps.

The results of this study demonstrate the successful detection of individual Varroa mites using accelerometers on multiple substrates and disclose a behaviour that is new to science, which we refer to as 'jolting'. The features of the associated vibrational pulse strongly indicate that it is possible to detect mites in honeybee hives using accelerometers, and this now requires further work to narrow down the location of pulse production for a more lucrative search. By exploring this, we may also shed light on the function of the pulse, as our early results tantalisingly suggest that the jolting behaviour is beneficial to the animal.

Acknowledgments: The authors wish to thank Nottingham Trent University for H. Hall PhD fellowship. We are grateful to Stephen Martin and Neil Mansfield for scientific and technical inputs, to Gard Otis for input on the manuscript, and to Yves Le Conte, Vincent Piou, Angélique Vétillard, Dirk de Graaf for scientific discussions on the topic.

\section{References}

Anderson, D. L., \& Trueman, J. W. H. (2000). Varroa jacobsoni (Acari: Varroidae) is more than one species. Experimental \& Applied Acarology, 24(3), 165-189. https://doi.org/10.1023/ A:1006456720416

Bąk, B., Wilk, J., Artiemjew, P., Wilde, J., \& Siuda, M. (2020). Diagnosis of varroosis based on bee brood samples testing with use of semiconductor gas sensors. Sensors (Basel), 20(14), 4014. https://doi.org/10.3390/s20144014

Barlow, V. M., \& Fell, R. D. (2006). Sampling methods for Varroa mites on the domesticated honeybee. Department of Entomology, Virginia Tech., US. Virginia Cooperative Extension. Publication. 444-103, 1-3.

Bencsik, M., Le Conte, Y., Reyes, M., Pioz, M., Whittaker, D., Crauser, D., ... Newton, M. I. (2015). Honeybee colony vibrational measurements to highlight the brood cycle. PLoS One, 10(11), e0141926. https://doi.org/10.1371/journal.pone.0141926

Bienkowska, M., \& Konopacka, Z. (2001). Assessment of honeybee colonies infestation by the mite Varroa destructor based on its natural mortality during the summer season. Journal of Apicultural Science, 45, 129-140.

Bilik, S., Kratochvila, L., Ligocki, A., Bostik, O., Zemcik, T., Hybl, M., ... Zalud, L. (2021). Visual Diagnosis of the Varroa Destructor Parasitic Mite in Honeybees Using Object Detector Techniques. Sensors (Basel), 21(8), 2764. https://doi.org/ $10.3390 / \mathrm{s} 21082764$

Bjerge, K., Frigaard, C. E., Mikkelsen, P. H., Nielsen, T. H., Misbih, M., \& Kryger, P. (2019). A computer vision system to monitor the infestation level of Varroa destructor in a honeybee colony. Computers and Electronics in Agriculture, 164, 104898. https:// doi.org/10.1016/j.compag.2019.104898 
Broad, G. R., \& Quicke, D. L. J. (2001). The adaptive significance of host location by vibrational sounding in parasitoid wasps. Proceedings of the Royal Society of London. Series B, Biological Sciences, 286(1484), 2403-2408.

Chazette, L., Becker, M., \& Szczerbicka, H. (2016). Basic algorithms for behive monitoring and laser-based mite control. In 2016 IEEE Symposium Series on Computational Intelligence (SSCI). 1-8.

Chen, Y. P., \& Siede, R. (2007). Honeybee viruses. Advances in Virus Research, 70, 33-80. https://doi.org/10.1016/S0065-3527 (07)70002-7

Dietemann, V., Nazzi, F., Martin, S. J., Anderson, D. L., Locke, B., Delaplane, K. S., ... Ellis, J. E. (2013). Standard methods for Varroa research. Journal of Apicultural Research, 52(1), 1-54. https://doi.org/10.3896/IBRA.1.52.1.09

Dillier, F., Fluri, P., \& Imdorf, A. (2006). Review of the orientation behaviour of the bee parasitic mite Varroa destructor: Sensory equipment and cell invasion behaviour. Revue Suisse de Zoologie, 113(4), 857-877. https://doi.org/10.5962/bhl.part. 80381

Donzé, G., \& Guerin, P. M. (1994). Behavioural attributes and parental care of Varroa mites parasitising honeybee brood. Behavioral Ecology and Sociobiology, 34(5), 305-319. https:// doi.org/10.1007/BF00197001

Dowling, A. (2015). The evolution of parasitism and host association in mites. In S. Morand, B. R. Krasnov, \& T. J. Littlewoord (Eds.), Parasite Diversity and Diversification (1 ${ }^{\text {st }}$ ed., pp. 265288). Cambridge: Cambridge University Press. https://doi. org/10.1017/CBO9781139794749.018

Elizondo, V., Briceno, J. C., Travieso, C. M., \& Alonso, J. B. (2013). Video monitoring of a mite in honeybee cells. Advanced Materials Research, 664, 1107-1113. https://doi.org/10.4028/ www.scientific.net/AMR.664.1107

Evans, J. D., \& Cook, S. C. (2018). Genetics and physiology of Varroa mites. Current Opinion in Insect Science, 26, 130-135. https://doi.org/10.1016/j.cois.2018.02.005

Fakhimzadeh, K., Ellis, J. D., \& Hayes, J. W. (2011). Physical control of Varroa mites (Varroa destructor): The effects of various dust materials on Varroa mite fall from adult honeybees (Apis mellifera). Journal of Apicultural Research, 50(3), 203-211. https://doi.org/10.3896/IBRA.1.50.3.04

Frey, E., Odemer, R., Blum, T., \& Rosenkranz, P. (2013). Activation and interruption of the reproduction of Varroa destructor is triggered by host signals (Apis mellifera). Journal of Invertebrate Pathology, 113(1), 56-62. https://doi.org/10.1016/j.jip.2013. 01.007

Gregorc, A., Knight, P. R., \& Adamczyk, J. (2017). Powdered sugar shake to monitor and oxalic acid treatments to control Varroa mites (Varroa destructor Anderson and Trueman) in honeybee (Apis mellifera) colonies. Journal of Apicultural Research, 56(1), 71-75. https://doi.org/10.1080/00218839.2017.1278912

Gregorc, A., \& Sampson, B. (2019). Diagnosis of Varroa mite (Varroa destructor) and sustainable control in honeybee (Apis mellifera) colonies - a review. Diversity (Basel), 11(12), 243. https://doi.org/10.3390/d11120243

Gronenberg, W. (1996). Fast actions in small animals: Springs and click mechanisms. Journal of Comparative Physiology. A, Neuroethology, Sensory, Neural, and Behavioral Physiology, 178(6), 727-734. https://doi.org/10.1007/BF00225821
Kevan, P. G., Hannan, M., \& Ostiguy, A. (2006). A summary of the varroa-virus disease complex in honeybees. American Bee Journal, 146(8), 694-697.

Kimchi, T., Reshef, M., \& Terkel, J. (2005). Evidence for the use of reflected self-generated seismic waves for spatial orientation in a blind subterranean mammal. The Journal of Experimental Biology, 208(4), 647-659. https://doi.org/10.1242/jeb.01396

Konig, A. (2021). First results of the be-e-nose on mid-term duration hive air monitoring for Varroa infestation level estimation. Sensors and Transducers, 250(3), 39-41.

Kriegbaum, H. (1997). Grasshopper reproductive strategies measured in the field: A tradeoff between age at maturity and egg production per day. Naturwissenschaften, 84(4), 157-159. https://doi.org/10.1007/s001140050370

Le Conte, Y., Ellis, M., \& Ritter, W. (2010). Varroa mites and honeybee health: Can varroa explain part of the colony losses? Apidologie, 41(3), 353-363. https://doi.org/10.1051/apido/ 2010017

Lee, K. V., Moon, R. D., Burkness, E. C., Hutchison, W. D., \& Spivak, M. (2010). Practical sampling plans for Varroa destructor (Acari: Varroidae) in Apis mellifera (Hymenoptera: Apidae) colonies and apiaries. Journal of Economic Entomology, 103(4), 1039-1050. https://doi.org/10.1603/EC10037

Markl, H. (1967). Die Verständigung durch Stridulationssignale bei Blattschneiderameisen. Zeitschrift für vergleichende Physiologie, 57(3), 299-330. https://doi.org/10.1007/BF00303001

Martin, S. (1998). A population model for the ectoparasitic mite Varroa jacobsoni inhoneybee (Apis mellifera) colonies. Ecological Modelling, 109(3), 267-281. https://doi.org/10.1016/ S0304-3800(98)00059-3

Martin, S. J. (2004). Acaricide (pyrethroid) resistance in Varroa destructor. Bee World, 85(4), 67-69. https://doi.org/10.1080/00 05772X.2004.11099632

Michelsen, A., \& Elsner, N. (1999). Sound emission and the acoustic far field of a signing acridid grasshopper (Omocestus viridulus L.). The Journal of Experimental Biology, 202(12), 1571-1577. https://doi.org/10.1242/jeb.202.12.1571

Michelsen, A., Fink, F., Gogala, M., \& Traue, D. (1982). Plants as transmission channels for insect vibrational songs. Behavioral Ecology and Sociobiology, 11(4), 269-281. https://doi.org/ 10.1007/BF00299304

Milani, N. (1999). The resistance of Varroa jacobsoni Oud. to acaricides. Apidologie, 30(2-3), 229-234. https://doi.org/10.1051/ apido:19990211

Nazzi, F., \& Le Conte, Y. (2016). Ecology of Varroa destructor, the major ectoparasite of the western honeybee, Apis mellifera. Annual Review of Entomology, 61(1), 417-432. https://doi. org/10.1146/annurev-ento-010715-023731

Otten, H., Wäckers, F., Battini, M., \& Dorn, S. (2001). Efficiency of vibrational sounding in the parasitoid Pimpla turionellae is affected by female size. Animal Behaviour, 61(3), 671-677. https://doi.org/10.1006/anbe.2000.1639

Patek, S. N., Dudek, D. M., \& Rosario, M. V. (2011). From bouncy legs to poisoned arrows: Elastic movements in invertebrates. The Journal of Experimental Biology, 214(12), 1973-1980. https://doi.org/10.1242/jeb.038596

Pettis, J. S. (2004). A scientific note on Varroa destructor resistance to coumaphos in the united states. Apidologie, 35(1), 91-92. https://doi.org/10.1051/apido:2003060 
Pietropaoli, M., Tlak Gajger, I., Costa, C., Gerula, D., Wilde, J., Adjlane, N., ... Formato, G. (2021). Evaluation of two commonly used field tests to assess Varroa destructor infestation on honeybee (Apis mellifera) colonies. Applied Sciences (Basel, Switzerland), 11(10), 4458. https://doi.org/10.3390/app11104458

Piou, V., Urrutia, V., Laffont, C., Hemptinne, J., \& Vetillard, A. (2019). The nature of the arena surface affects the outcome of host-finding behaviour bioassays in Varroa destructor (Anderson and Trueman). Parasitology Research, 118(10), 2935-2943. https://doi.org/10.1007/s00436-019-06435-2

Ramirez, M., Prendas, J. P., Travieso, C. M., Calderon, R., \& Salas, O. (2012). Detection of the mite Varroa destructor in honeybee cells by video sequence processing. In 2012 IEEE $16^{\text {th }}$ International Conference on Intelligent Engineering Systems (INES). 103-108.

Ramsey, M., Bencsik, M., \& Newton, M. I. (2018). Extensive vibrational characterisation and long-term monitoring of honeybee dorso-ventral abdominal vibration signals. Scientific Reports, 8, 14571. https://doi.org/10.1038/s41598-018-32931-z

Ramsey, M., Bencsik, M., \& Newton, M. I. (2017). Long-term trends in the honeybee whooping signal revealed by automated detection. PLoS One, 12(7), e0181736. https://doi.org/10.1371/ journal.pone.0181736

Ramsey, M., Bencsik, M., Newton, M. I., Reyes, M., Pioz, M., Crauser, D., ... Le Conte, Y. (2020). The prediction of swarming in honeybee colonies using vibrational spectra. Scientific Reports, 10, 9798. https://doi.org/10.1038/s41598-020-66115-5

Ramsey, S. D., Ochoa, R., Bauchan, G., Gulbronson, C., Mowery, J. D., Cohen, A., ... van Engelsdorp, D. (2019). Varroa destructor feeds primarily on honeybee fat body tissue and not haemolymph. Proceedings of the National Academy of Sciences of the United States of America, 116(5), 1792-1801. https://doi. org/10.1073/pnas.1818371116

Ramsier, M. A., \& Dominy, N. J. (2012). Receiver bias and the acoustic ecology of aye-ayes (Daubentonia madagascariensis). Communicative \& Integrative Biology, 5(6), 637-640. https:// doi.org/10.4161/cib.21509

Reeping, D., Reid, K. (2016). Introductory Engineering Mathematics. Momentum Press. 149-152.

Roces, F., \& Holldobler, B. (1994). Leaf density and a trade-off between load-size selection and recruitment behaviour in the ant Atta cephalotes. Oecologia, 97, 1-8. https://doi.org/10.1007/ BF00317902

Rosenkranz, P., Aumeier, P., \& Ziegelmann, B. (2010). Biology and control of Varroa destructor. Journal of Invertebrate Pathology, 103, S96. https://doi.org/10.1016/j.jip.2009.07.016

Schmolz, E., Kosece, F., \& Lamprecht, I. (2005). Energetics of honeybee development. Thermochimica Acta, 437(1-2), 39-47. https://doi.org/10.1016/j.tca.2005.06.001

Sterling, E. J., \& McCreless, E. E. (2006). Adaptations in the ayeaye: a review. Lemurs. 159-184.

Szczurek, A., Maciejewska, M., Bąk, B., Wilk, J., Wilde, J., \& Siuda, M. (2020a). Gas sensor array and classifiers as a means of varroosis detection. Sensors (Basel), 20(1), 117. https://doi. org/10.3390/s20010117

Szczurek, A., Maciejewska, M., Zajiczek, Z., Bąk, B., Wilk, J., Wilde, J., \& Siuda, M. (2020b). The effectiveness of Varroa destructor infestation classification using an e-nose depending on the time of day. Sensors (Basel), 20(9), 2532. https://doi. org/10.3390/s20092532

Tihelka, E. (2018). Effects of synthetic and organic acaricides on honeybee health: A review. Slovenian Veterinary Research, 55(3), 119-140. https://doi.org/10.26873/SVR-422-2017

Wäckers, F. L., Mitter, E., \& Dorn, S. (1998). Vibrational sounding by the pupal parasitoid Pimpla (Coccygomimus) turionellae: An additional solution to the reliability-detectability problem. Biological Control, 11(2), 141-146. https://doi.org/10.1006/ bcon. 1997.0592

Ziegelmann, B., Lindenmayer, A., Steidle, J., \& Rosenkranz, P. (2012). The mating behaviour of Varroa destructor is triggered by a female sex pheromone. Apidologie, 44(3), 314-323. https:// doi.org/10.1007/s13592-012-0182-5

Manuscript received: 28 July 2021

Revisions requested: 10 August 2021

Modified version received: 28 October 2021

Accepted: 16 November 2021

The supplementary material to this article is deposited at https://zenodo.org

https://doi.org/10.5281/zenodo.5616929 = s1 https://doi.org/10.5281/zenodo.5616977 = s2 https://doi.org $/ 10.5281 /$ zenodo.5617016 = s3 https://doi.org/10.5281/zenodo.5617026 = s4 https://doi.org $/ 10.5281 /$ zenodo.5617046 = s5 https://doi.org/10.5281/zenodo. $5617070=\mathrm{s} 6$ https://doi.org $/ 10.5281 /$ zenodo. $5617093=\mathrm{s} 7$ https://doi.org/10.5281/zenodo.5617163 = s8 https://doi.org/10.5281/zenodo.5617205 = s9 https://doi.org/10.5281/zenodo.5617231 = s10 https://doi.org/10.5281/zenodo.5617253 = s11 https://doi.org/10.5281/zenodo.5617271 = s12 https://doi.org/10.5281/zenodo.5617321 = s13 https://doi.org/10.5281/zenodo.5617365 = s14 https://doi.org/10.5281/zenodo.5617390 = s15 https://doi.org/10.5281/zenodo.5617410 = s16 https://doi.org/10.5281/zenodo.5617425 = s17 https://doi.org/10.5281/zenodo.5617457 = s18 https://doi.org/10.5281/zenodo.5617469 = s19 https://doi.org/10.5281/zenodo.5617499 = s20 https://doi.org/10.5281/zenodo.5617528 = s21 https://doi.org/10.5281/zenodo.5617542 = s22 https://doi.org/10.5281/zenodo.5617556 = s23 https://doi.org/10.5281/zenodo.5617576 = s24 https://doi.org/10.5281/zenodo.5617592 = s25 https://doi.org/10.5281/zenodo.5617622 = s26 https://doi.org/10.5281/zenodo.5617638 = s27 https://doi.org/ $10.5281 /$ zenodo. $5617648=\mathrm{s} 28$ https://doi.org/10.5281/zenodo.5617670 = s29 https://doi.org/10.5281/zenodo.5617690 = s30 https://doi.org/10.5281/zenodo.5617706 = s31 